\title{
On the Design of Aircraft Electrical Structure Networks
}

\author{
Guadalupe G. Gutierrez, Daniel Mateos Romero, Miguel Ruiz Cabello, Enrique Pascual-Gil, Member, IEEE, \\ Luis Diaz Angulo, and Salvador Gonzalez Garcia, Senior Member, IEEE
}

\begin{abstract}
As part of the technology research engaged in the EU Clean Sky 1 project, we present in this paper an electrical structure network (ESN) designed to prevent the impact on an electronic equipment of unwanted voltage drops appearing when nonmetal composite materials are used for grounding. An iterative process has been followed to reach an optimal tradeoff solution meeting all the aircraft requirements: structural, safety, low weight, electrical, etc. Guidelines on the design of a low-impedance metal ESN, to minimize the inductive behavior of the power distribution network, are outlined in this paper. To this end, we employ the UGRFDTD simulation tool, combining finite-difference time domain to analyze the general EM problem, and a multiconductor transmission-line network to handle internal coupling between cables running along coinciding routes. The capability of this tool to create time-domain snapshots of surface currents is shown to provide a useful way to optimize the ESN, thanks to the insight gained on the physics of the problem.
\end{abstract}

Index Terms-Carbon-fiber-reinforced plastic (CFRP), electrical structure network, electromagnetic compatibility (EMC), finite-difference time domain (FDTD), green regional aircraft (GRA).

\section{INTRODUCTION}

$\mathbf{T}$ HE design of an aircraft (A/C) must comply with the aerodynamic, structural, safety, fuel efficiency, cost, and electromagnetic (EM) requirements, among many others, this requiring the parallel work of several departments. Clean Sky 1 is one of the largest European R\&D projects focused on the design of the $\mathrm{A} / \mathrm{C}$ of the future: low- $\mathrm{CO}_{2}$ and highly cost-efficient air-transport system. Its objective is to speed up the technological breakthrough developments and to shorten the time-to-market for new solutions that introduce green technology into aviation. Within the framework of Clean Sky 1 , this paper deals with the design of low-weight configurations

Manuscript received May 27, 2015; revised October 26, 2015; accepted January 1, 2016. This work was supported from the European Community Seventh Framework Program FP7/2008-2014 under Grant CSJU-GAM-GRA2008-01 (Clean Sky 1 project), and from the Projects TEC2013-48414-C301 and TEC2015-68766-REDC (MINECO, Spain), P12-TIC-1442 (Junta de Andalucia, Spain), Alhambra-UGRFDTD (AIRBUS DS), and by the CSIRC alhambra.ugr.es supercomputing center.

G. G. Gutiérrez and E. Pascual-Gil are with Department of Airbus Defence and Space, Getafe 28906, Spain (e-mail: Guadalupe.Gutierrez@airbus.com; Enrique.Pascual@airbus.com).

D. Mateos, M. R. Cabello, L. D. Angulo, and S. G. Garcia are with the Department of Electromagnetism, University of Granada, Granada 18071, Spain (e-mail: salva@ugr.es; damarro@ugr.es; mcabello@ugr.es; lmdiazangulo@ ugr.es).

Color versions of one or more of the figures in this paper are available online at http://ieeexplore.ieee.org.

Digital Object Identifier 10.1109/TEMC.2016.2514379 to reduce the fuel consumption, meeting EM requirements for the effective protection of equipment, in terms of grounding.

This technology is to be integrated into the so-called green regional aircraft (GRA), which is one of the integrated technology demonstrators of the Clean Sky project. The GRA must deliver a low-weight A/C using smart structures, as well as a low external noise configuration, and it must also integrate the technology developed in other integrated technology demonstrators, such as engines, energy management, and new system architecture [1].

The increased use of carbon-fiber-reinforced plastics (CFRP) in the aeronautic industry has led to several advantages in terms of weight and cost, but entails other disadvantages related to its EM performance. A major drawback is poorer conductivity compared with the traditional metal materials, causing different pieces of equipment grounded at different points of a CFRP not to operate with the same ground reference. These differences may be significant enough to impact electronic designs.

The use of an electrical structure network (ESN) provides a solution to endow the structure with an adequate current return path, and effective protection against EM-induced effects. ${ }^{1}$ An ESN typically consists of a distributed metal network, with negligible resistance, whose inductive impedance must be kept low enough to ensure a quasi-equipotential ground reference for the different pieces of equipment [2], [3].

In this paper, we focus on the design of an ESN for equipment and corresponding power-line wiring located in the cockpit zone, having a development assurance level either critical for safety (A), or hazardous (B). We present guidelines for the design of a low-impedance ESN for the protection of equipment under $1 \mathrm{MHz}$, enforcing a low-weight requirement so as not to jeopardize the goal of developing nonmetal $\mathrm{A} / \mathrm{C}$ lighter than their metal counterparts. For this, we focus on the reduction of the inductive behavior of the power distribution network, since inductance is responsible for worsening the quality of the electrical power arriving to the equipment, by creating a voltage noise at frequencies usually present on airborne electronic systems [2], [3]. Equipment operation is challenged by voltage ripples at power input and voltage reference; thus, reducing the reliability of the signals processed by the electronic components. Furthermore, any significant inductance on ground reference precludes the mitigation of radio-frequency noise compromising the EM Compatibility of the electric and electronic systems of the A/C.

\footnotetext{
${ }^{1}$ ESN-related topics (almost equipotential network, current return networks) have also been addressed within some recent FP7 EU projects like GENIAL or ARROW.
} 
For our purpose, computational electromagnetic (CEM) tools have been used, for their proven capacity of making parametrical studies to help during the design phase, when changes are simpler and less costly, and to cut down on the costs and risks of experimental tests. Simulation tools based on full-wave time-domain numerical methods to solve the Maxwell equations are especially useful for their generality and broadband nature. Among them, the finite-difference time-domain (FDTD) method [4], [5] has become one of the most widely used in CEM. During the recent EU FP7 HIRF-SE project [6] Airbus Defence and Space and the University of Granada (Spain) collaborated in the development and validation of the University of Granada Finite Difference Time Domain (UGRFDTD) parallel 3-D solver [7] for the assessment of high-intensity radiated fields (HIRF) effects in full A/C. UGRFDTD makes use of the FDTD to address the general EM problem, combined with a thin-wire [8] multiconductor transmission-line network (MTLN) algorithm taking into account the coupling between the different cables and bundles running along coinciding routes [9], and thus suitable to deal with the typically complex layout of the A/C power-line networks under study in this paper.

The UGRFDTD tool has proven to be highly suitable to deal with the EMC problems of HIRF/Lightning/nuclear electromagnetic pulse involving complex and electrically large geometries [10]-[12]. Its capability to provide field and current snapshots compatible with the paraview [13] visualization tool is shown in this paper to be a valuable asset to help in the design and analysis of the ESN topologies.

It bears stressing that efficient CEM tools alone may not be enough to achieve our goal [14]. Computationally affordable numerical models of the objects under test, yet sufficiently clean and simplified, retaining at the same time all the relevant EM physical information through proper approximations, are required. The availability of exact $\mathrm{CAD}$ and material data and the experience of personnel performing these tasks is crucial to reduce the uncertainties of the results [10]-[12], [15]-[17].

For this reason, we employ in this paper, a well-controlled model for a C-295 A/C, already used for the UGRFDTD validation under HIRF-SE [12]. For this model, we establish in an iterative manner a so-called optimized solution for the ESN topology, in terms of electrical performance. This solution is further modified to comply with all the requirements of lifecycle maintenance and bird-impact safety, proposed by other departments involved in the A/C design, to reach a final ESN, which has been analyzed with respect to the optimized solution, and finally proposed and adopted for its implementation in the GRA.

\section{NUMERICAL SOLVER}

The UGRFDTD parallel 3-D solver [7] can employ either a conformal [18] or a classical Yee staircased spatial mesh [4]. In this paper, a staircased mesh of the C-295 A/C, already validated under HIRF-SE [6], has been employed. The usual leap-frog FDTD marching-on-in-time scheme, with the electric fields at semiinteger multiples of the time increment $\Delta t$ and the magnetic fields at integer multiples for the staircased mesh, can be written as [19]:

$$
\begin{aligned}
\mathbf{E}_{i, j, k}^{n+1 / 2}= & \frac{\varepsilon_{i, j, k}-\sigma_{i, j, k} \Delta t / 2}{\varepsilon_{i, j, k}+\sigma_{i, j, k} \Delta t / 2} \mathbf{E}_{i, j, k}^{n-1 / 2} \\
& +\frac{\Delta t}{\varepsilon_{i, j, k}+\sigma_{i, j, k} \Delta t / 2} \tilde{\delta}_{r} \mathbf{H}_{i, j, k}^{n} \\
\mathbf{H}_{i, j, k}^{n+1}= & \frac{\mu_{i, j, k}-\sigma_{i, j, k}^{*} \Delta t / 2}{\mu_{i, j, k}+\sigma_{i, j, k}^{*} \Delta t / 2} \mathbf{H}_{i, j, k}^{n} \\
& -\frac{\Delta t}{\mu_{i, j, k}+\sigma_{i, j, k}^{*} \Delta t / 2} \tilde{\delta}_{r} \mathbf{E}_{i, j, k}^{n+1 / 2}
\end{aligned}
$$

with $n$ integer, and $i, j, k$ integers or semiintegers according to the position of the field component at the Yee cell, and the numerical curl operator $\tilde{\delta_{r}}$ :

$$
\begin{aligned}
\tilde{\delta_{r}} & =\left(\begin{array}{ccc}
0 & -\delta_{z} & \delta_{y} \\
\delta_{z} & 0 & -\delta_{x} \\
-\delta_{y} & \delta_{x} & 0
\end{array}\right) \\
\delta_{v} f(v) & =\frac{f\left(v+\frac{\Delta v}{2}\right)-f\left(v-\frac{\Delta v}{2}\right)}{\Delta v} .
\end{aligned}
$$

We combine this scheme with material independent complexfrequency-shifted perfectly matched layer [20] truncation boundary conditions, which in the frequency domain can be written as

$$
-\tilde{\delta}_{r} \mathbf{E}_{i, j, k}=\mathrm{j} \omega \tilde{s} \mathbf{B}_{i, j, k}, \tilde{\delta}_{r} \mathbf{H}_{i, j, k}=\mathrm{j} \omega \tilde{s} \mathbf{D}_{i, j, k}
$$

with

$$
\tilde{s}=\left(\begin{array}{ccc}
\frac{s_{y} s_{z}}{s_{x}} & 0 & 0 \\
0 & \frac{s_{z} s_{x}}{s_{y}} & 0 \\
0 & 0 & \frac{s_{x} s_{y}}{s_{z}}
\end{array}\right), s_{\alpha}=\kappa_{\alpha}+\frac{\sigma_{\alpha}}{\eta_{\alpha}+\mathrm{j} \omega \chi_{\alpha}}
$$

and implemented in time domain using a convolutional form [21].

A MTLN thin-wire approach [9] is hybridized with the previous FDTD scheme, to deal with cables sharing the common edges of Yee-FDTD cells. For instance, along the $z$-direction

$$
\partial_{z} \mathbf{I}+\tilde{C} \partial_{t} \mathbf{V}=0, \tilde{R} \mathbf{I}+\tilde{L} \partial_{t} \mathbf{I}+\partial_{z} \mathbf{V}=\left\langle\mathbf{E}_{z}\right\rangle
$$

where $\mathbf{I}$ and $\mathbf{V}$ hold the vector with the currents and voltages at each segment sharing the path, $\tilde{R}, \tilde{L}, \tilde{C}$ are the p.u.l. resistance, inductance, and capacitance MTLN matrices found by using the technique described in [9], while $\left\langle\mathbf{E}_{z}\right\rangle$ is a vector with the average $z$-component of the electric field along each wire.

The whole algorithm is parallelized with a mixed OpenMP/MPI methodology [22], scaling in typical infinibandbased clusters with speeds up to $20 \mathrm{Mcells} / \mathrm{s} /$ core.

\section{Simulation Setup}

A digital mock-up of the complete cockpit of the C-295 A/C, already cleaned and simplified following the methodology described in [12] and [23], has been used. For the purpose of this 
TABLE I

LIST OF EQUIPMENT UNDER TEST (EUT), LISTED WiTH RESPECT TO THEIR DEVELOPMENT ASSURANCE LEVEL (DAL): CRITICAL FOR SAFETY (A) AND HAZARDOUS (B)

\begin{tabular}{lcc}
\hline \hline EUT & Equipment Description & DAL \\
\hline EFIS-ND & Electronic Flight Instrument System-Navigation Display & $\mathrm{A}$ \\
EFIS PFD & Electronic Flight Instrument System-Primary Flight Display & $\mathrm{A}$ \\
EFIS ICP & Electronic Flight Instrument System-Index Control Panel & $\mathrm{A}$ \\
ADU & Air Data Unit & $\mathrm{A}$ \\
IFC-IOP & Integrated Flight Cabinets-Input Output Processor & $\mathrm{A}$ \\
IFF & Identification Friend/Foe & $\mathrm{B}$ \\
MCDU & Multifunctional Control Display Unit & $\mathrm{B}$ \\
IEDS & Integrated Engine Display System & $\mathrm{B}$ \\
CEU & Control Engagement Unit & $\mathrm{B}$ \\
FECU & Flap Electronic Control Unit & $\mathrm{B}$ \\
\hline \hline
\end{tabular}

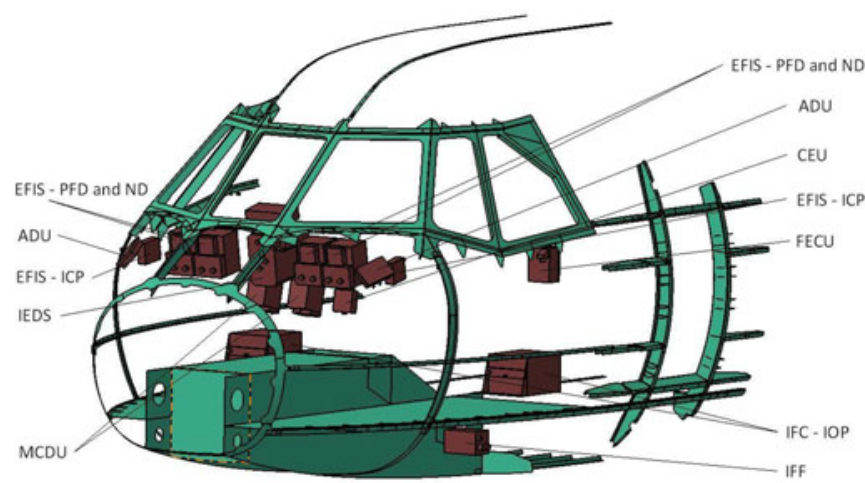

Fig. 1. Location of equipment listed in Table I.

paper, we have included in the model only the critical and hazardous equipment (listed in Table I and shown in Fig. 1), and modeled in this paper as 50- $\Omega$ loads between their power lines and their grounding points.

A voltage source has been used to excite the power lines connected to the electrical panels, assumed to be perfect electrical conductor (PEC), situated in the cockpit, using the ESN as ground reference. Two different configurations according to the source location have been analyzed, one of them with the source applied at the right side and the other one with the source at the left side (see Fig. 6), so as to take into account the cable route asymmetry present in the model. For the sake of simplicity, only the results for the right-sided source are presented, since the other configuration adds no new conclusion.

A Gaussian waveform with few megahertz of spectral content [24] has been used to excite the PEC plate, given by

$$
f(t)=28 e^{-\alpha\left(t-t_{0}\right)^{2}}, \alpha=2 \cdot 10^{13} s^{-2}, t_{0}=10^{-6} \mathrm{~s} .
$$

This model has been meshed in a Cartesian grid, suitable for the FDTD, with a constant space-step $\Delta=10 \mathrm{~mm}$, yielding a problem size of $\sim 83 \mathrm{M}$ cells. A time step of $\Delta t=8 \mathrm{ps}$ has been employed to meet the Courant-Friedrichs-Lewy stability condition [5], and that imposed by the thin-wire approach [25]. A total time of $10 \mu$ s has been simulated for having proven to be sufficient to have a fair convergence on the currents. Each simulation has taken around one day of CPU in an OpenMPMPI parallel architecture (64 threads running in 4 nodes, each

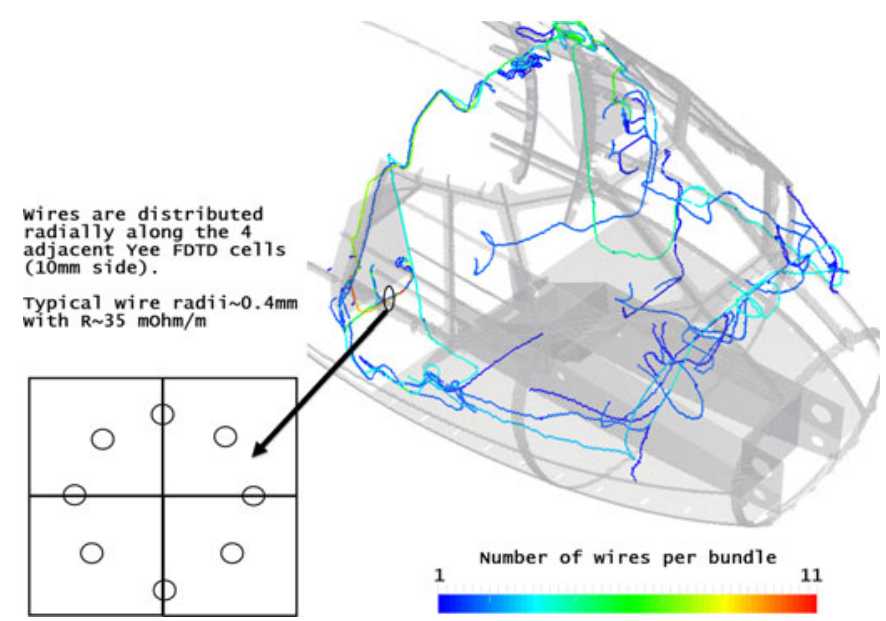

Fig. 2. Typical cross section of cable bundles.

TABLE II

CONFIGURATION DESIGNATIONS

\begin{tabular}{|c|c|c|}
\hline Name & Configuration & Description \\
\hline S & Starting guess & $\begin{array}{l}\text { Isolated ESN designed by only taking into account } \\
\text { structural requirements }\end{array}$ \\
\hline $\mathrm{R}$ & Reference & $\begin{array}{l}\mathrm{S} \text { config. enclosed in a PEC model of all the } \\
\text { aerodynamic surfaces in the original A/C }\end{array}$ \\
\hline $\mathrm{E}$ & Enhanced model & $\begin{array}{l}\text { S config. with additional ring-shaped and } \\
\text { stringer-shaped strips }\end{array}$ \\
\hline $\mathrm{O}$ & Optimized model & $\begin{array}{l}\mathrm{S} \text { config. with additional strips parallel to the cable } \\
\text { routes }\end{array}$ \\
\hline $\mathrm{F}$ & Final solution & $\begin{array}{l}\text { Final solution including feedback of requirements from } \\
\text { different departments }\end{array}$ \\
\hline
\end{tabular}

one with 2 Xeon E5-2680 8-core processors), 6.6-Gb memory (double-precision reals).

The power lines of equipment have been modeled as thin wires, treated with the MTLN approach given in [9] in order to ensure more accurate results (see Fig. 2). Equipment current return paths to the ESN are contained in the model as well. For that purpose, those current return cables which were connected with the original C-295 metal surfaces, but which cannot be attached to the corresponding point at the GRA (because they fall on a CFRP part), have been lengthened to the closest ESN surface.

A set of current probes at each current return path have been recorded in time, and transformed into frequency, to estimate the network impedance $Z$ by dividing it by the voltage source. From its imaginary part, its self-inductance is found by $L=$ $\Im m\{Z\} / 2 \pi f[26]$.

The iterative process, starting from pure structural requirements, and ending up in an ESN model that fulfills all the requirements, is described below.

\section{ESN DESIGN: AN ITERATIVE APPROACH}

Five different configurations have been simulated and analyzed in order to define an adequate ESN (presented in Table II). 


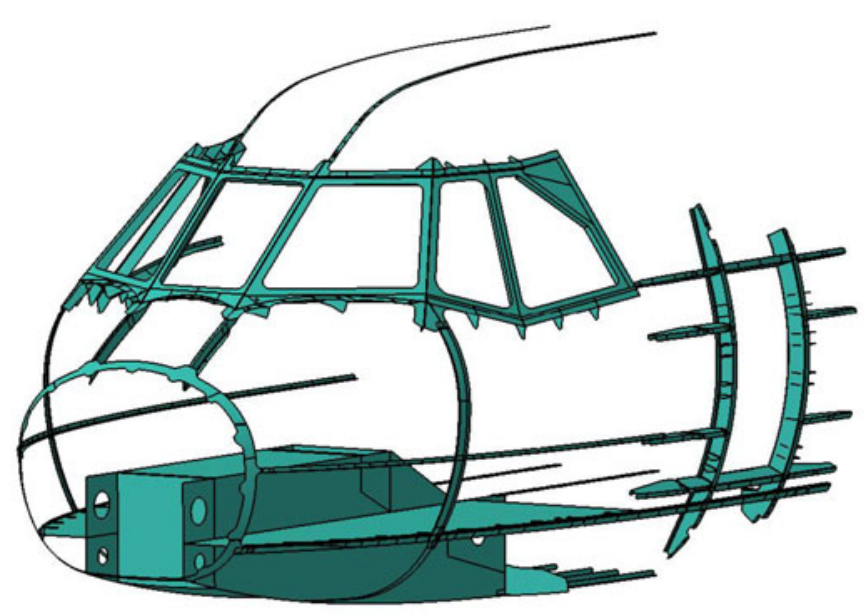

Fig. 3. Starting ESN, just fulfilling structural requirements.

TABLE III

L (IN H UNITS) FOR THE STARTING CONFIGURATION

\begin{tabular}{lc}
\hline \hline Equipment & \multicolumn{1}{c}{ S Conf. } \\
\hline EFIS-ND & $3.2602 \mathrm{E}-05$ \\
EFIS-PFD & $9.5914 \mathrm{E}-05$ \\
EFIS-ICP & $5.3054 \mathrm{E}-05$ \\
ADU & $2.0337 \mathrm{E}-04$ \\
IFC-IOP & $2.4930 \mathrm{E}-05$ \\
IFF & $2.2848 \mathrm{E}-04$ \\
MCDU & $3.9371 \mathrm{E}-05$ \\
IEDS-1A-1.2 & $7.4688 \mathrm{E}-04$ \\
IEDS-1A-3.4 & $3.0530 \mathrm{E}-05$ \\
IEDS-1B-1.2 & $8.9484 \mathrm{E}-05$ \\
IEDS-1B-3.4 & $2.4702 \mathrm{E}-05$ \\
CEU & $5.2537 \mathrm{E}-05$ \\
FECU & $6.7650 \mathrm{E}-05$ \\
\hline \hline
\end{tabular}

\section{A. Structural Requirements: ESN Starting Guess (S)}

Initially, the ESN was designed by taking into account only the input from departments imposing structural requirements. All aerodynamic surfaces were removed from the model since they are made of CFRP. Then, this starting ESN is composed of the metal elements depicted in Fig. 3 and it has been simulated yielding the results for $L$ presented in Table III.

\section{B. ESN Starting Model Inside a PEC Cockpit: Reference $(R)$}

We have also simulated a fully metal PEC model of the cockpit surrounding the initial guess for the ESN. We will refer to this case as a reference solution (R) in the sense that the PEC cockpit will provide, for being ohmically connected to the ESN, the best solution to conduct the return currents along paths of minimum impedance, as expected. ${ }^{2}$

The inductance of cables inside the original A/C (see Fig. 4), assuming that all metal aerodynamic surfaces are PEC, has been evaluated. Table IV shows the $L$ values found for the equipment

\footnotetext{
${ }^{2}$ Remember that for the GRA cockpit, the CFRP material will not permit such a perfect flow of return currents, which is one of the reasons for needing an ESN.
}

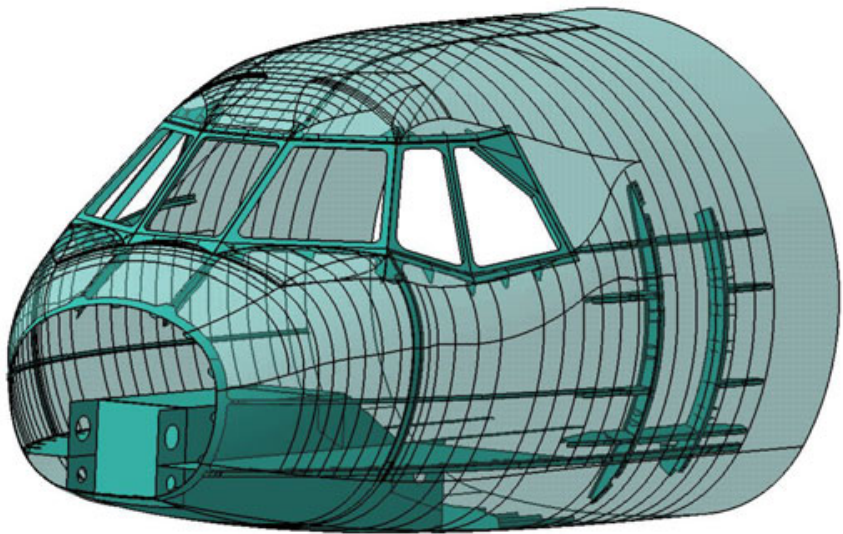

Fig. 4. Model with PEC aerodynamic surfaces (aerodynamic surfaces are depicted with a degree of transparency to also visualize the initial guess for the ESN of Fig. 3).

TABLE IV

L (IN H UNITS) FOR THE REFERENCE PEC CONFIGURATION AND PERCENTAGES OF DiFFERENCES With RESPECT TO THE STARTING ESN MODEL

\begin{tabular}{lcc}
\hline \hline Equipment & R Conf. & $\%(\mathrm{~S}-\mathrm{R})$ \\
\hline EFIS-ND & $2.6422 \mathrm{E}-05$ & 23 \\
EFIS-PFD & $6.7388 \mathrm{E}-05$ & 42 \\
EFIS-ICP & $3.9375 \mathrm{E}-05$ & 35 \\
ADU & $1.6188 \mathrm{E}-04$ & 26 \\
IFC-IOP & $2.1360 \mathrm{E}-05$ & 17 \\
IFF & $2.1753 \mathrm{E}-04$ & 5 \\
MCDU & $3.0544 \mathrm{E}-05$ & 29 \\
IEDS-1A-1,2 & $1.9116 \mathrm{E}-04$ & 29 \\
IEDS-1A-3,4 & $2.5800 \mathrm{E}-05$ & 18 \\
IEDS-1B-1,2 & $6.5511 \mathrm{E}-05$ & 37 \\
IEDS-1B-3,4 & $2.0961 \mathrm{E}-05$ & 18 \\
CEU & $4.8712 \mathrm{E}-05$ & 8 \\
FECU & $3.8964 \mathrm{E}-05$ & 74 \\
\hline \hline
\end{tabular}

under study for this configuration. As expected, $L$ obtained from this reference model with metal aerodynamic surfaces are lower than those of the starting case, and, actually, the minimum ones among all the ESN configurations studied in this paper.

\section{Adding Strips to the ESN: Enhanced Model (E)}

The first intuitive attempt to reduce the self-inductance of the starting guess ESN and, at the same time, maintaining the cockpit aerodynamic surfaces made of carbon fiber, is to design a denser metal ESN, with higher optical coverage. Fig. 5 shows the model with the addition of several ring-shaped and stringershaped strips.

Table $\mathrm{V}$ shows the $L$ values found for the equipment under study for this configuration and the percentage of difference with regard to the starting ESN and the reference case. As can be seen there, $L$ values for this new ESN design are in most cases smaller; thus, improving the performance of the starting ESN configuration, while they are greater than those of the metal reference case. Therefore, the use of a more dense ESN seems to be advisable, at the cost of an increased weight. 


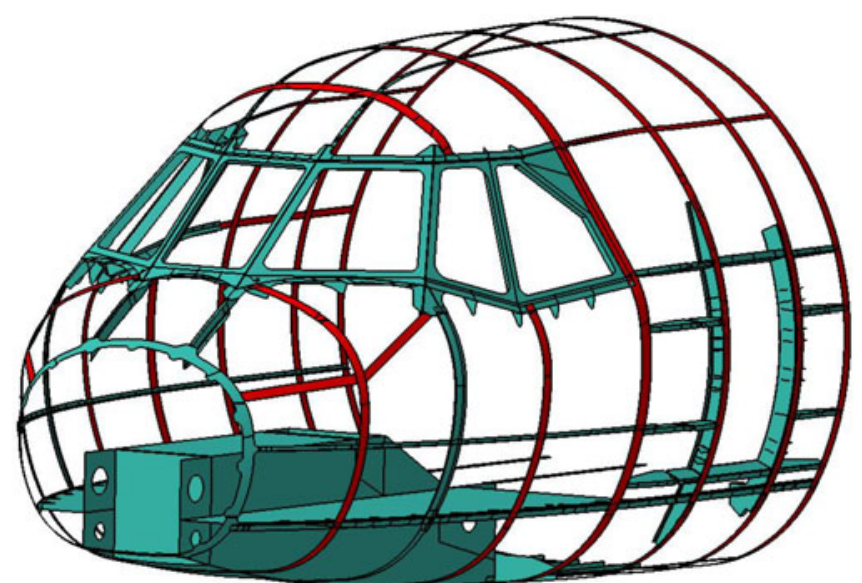

Fig. 5. Model with additional ring-shaped and stringer-shaped strips in red.

TABLE V

L (IN H UNITS) FOR THE ENHANCED CONFIGURATION AND PERCENTAgeS OF DIFFERENCES WITH RESPECT TO PREVIOUS ONES

\begin{tabular}{lccc}
\hline \hline Equipment & E Conf. & $\%(\mathrm{E}-\mathrm{R})$ & $\%(\mathrm{E}-\mathrm{S})$ \\
\hline EFIS-ND & $2.8835 \mathrm{E}-05$ & 9 & -13 \\
EFIS-PFD & $7.1829 \mathrm{E}-05$ & 7 & -34 \\
EFIS-ICP & $4.5182 \mathrm{E}-05$ & 15 & -17 \\
ADU & $1.7308 \mathrm{E}-04$ & 7 & -18 \\
IFC-IOP & $2.1874 \mathrm{E}-05$ & 2 & -14 \\
IFF & $2.4958 \mathrm{E}-04$ & 15 & 8 \\
MCDU & $3.3236 \mathrm{E}-05$ & 9 & -18 \\
IEDS-1A-1.2 & $3.5569 \mathrm{E}-04$ & 86 & -11 \\
IEDS-1A-3.4 & $2.7477 \mathrm{E}-05$ & 7 & -11 \\
IEDS-1B-1.2 & $7.4660 \mathrm{E}-05$ & 14 & -20 \\
IEDS-1B-3.4 & $2.2155 \mathrm{E}-05$ & 6 & -11 \\
CEU & $5.2303 \mathrm{E}-05$ & 7 & 0 \\
FECU & $4.2213 \mathrm{E}-05$ & 8 & -60 \\
\hline \hline
\end{tabular}

\section{ESN Following Surface Current Paths: Optimized}

\section{$\operatorname{Model}(O)$}

The increased weight of the above solution led us to search for a procedure to design an adequate ESN, based on the analysis of the paths followed by the currents in the original full PEC. Therefore, we have taken advantage of UGRFDTD ability to provide picture and video plots with electric surface current densities both in the time and in the frequency domain. By making use of these plots, we can determine the paths chosen by the current to flow along the structure at selected time steps during the threat, or at the frequencies of interest.

Snapshots with the electric surface-current density at a time of $1 \mu$ s have been used to perform the aforementioned analysis. Fig. 6 shows that the current flows along the aerodynamic surfaces roughly following the cable routes. The current tries to find the lowest inductance return path, by flowing through the zones of the PEC surface comprising the lowest loop area with the cables.

This observation led us to build an ESN by adding to the starting ESN (required for structural requirements) metal strips along the current paths, near cable routes, which is shown to be what we will call an optimized solution (see Fig. 7).
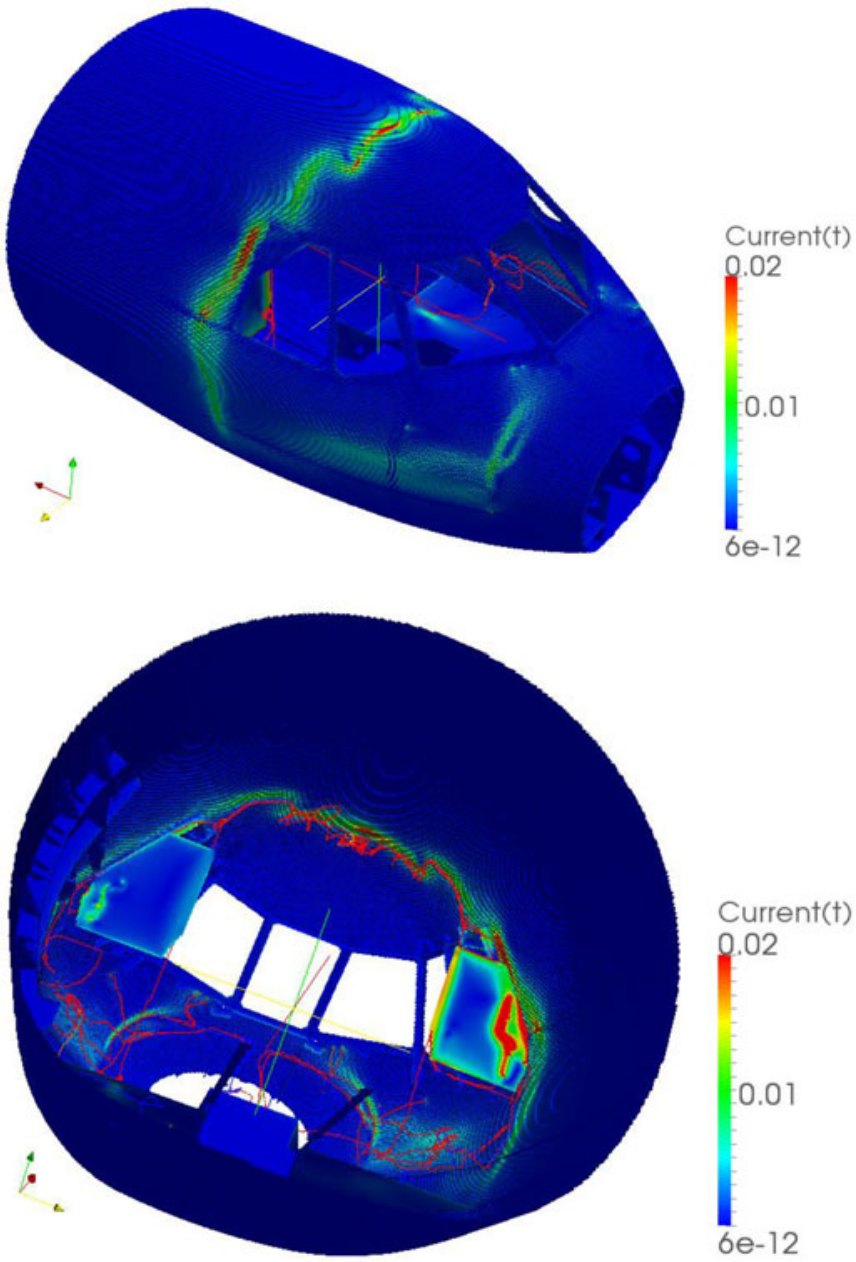

Fig. 6. Time snapshot of the electric surface current density on the C-295 metal cockpit with a voltage source on the right side. (a) Outside view). (b) Inside view.

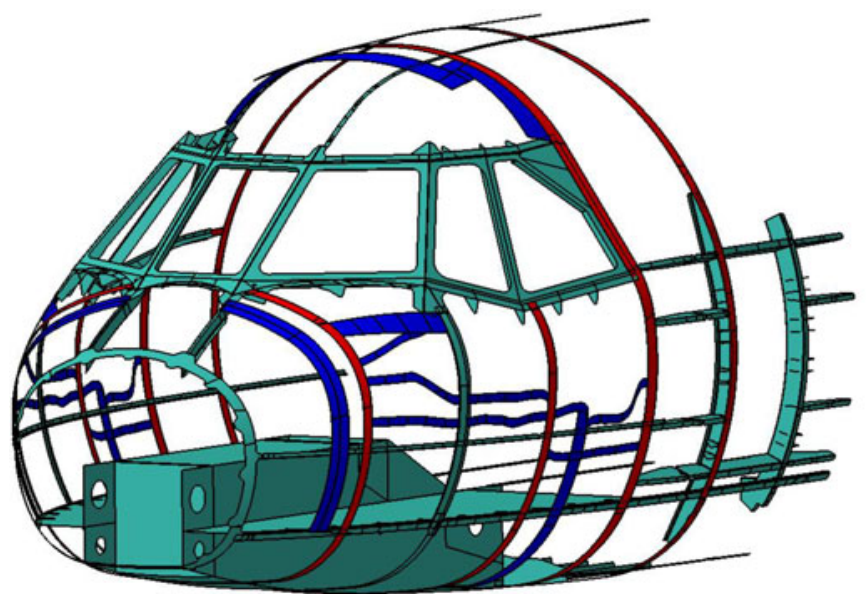

Fig. 7. Model with additional strips parallel to the cable routes in dark blue.

Time-domain results (see Fig. 8 and Table VI) show the delay between the current and the voltage (voltage peak is at $1 \mu \mathrm{s}$ ) due to the network inductance. It becomes apparent that, in general, the delay of the optimized configuration $(\mathrm{O})$ is similar to the 


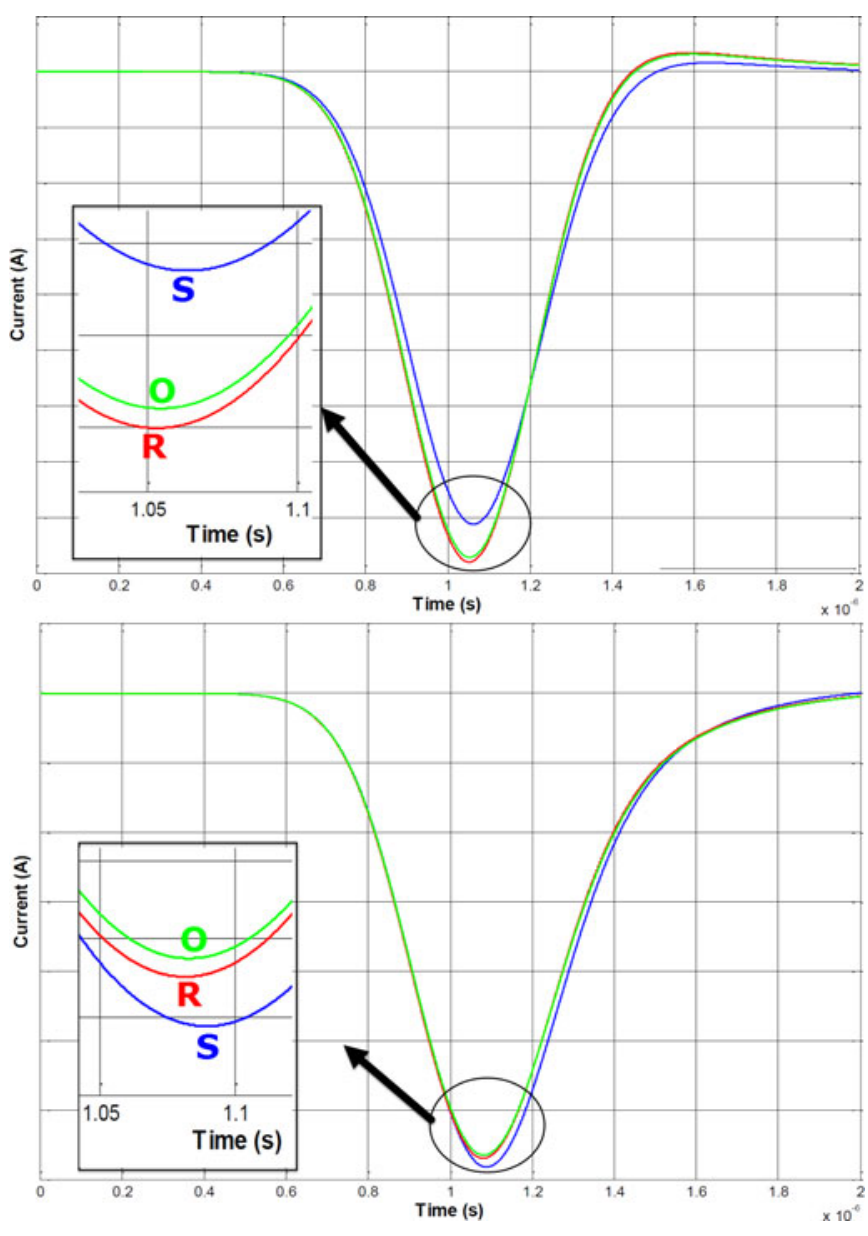

Fig. 8. Current at EFIS-ND (upper) and EFIS-PFD (lower) return paths for the $\mathrm{O}$ model, compared to configurations $\mathrm{S}$ and $\mathrm{R}$ (a detailed view is shown in the inset). The vertical scale has been removed due to nondisclosure and intellectual-property rights.

TABLE VI

DELAY TIMES (IN $\mu$ S) FOR THE CURRENT IN THE OPTIMIZED CONFIGURATION WITH RESPECT TO PREVIOUS ONES

\begin{tabular}{lccc}
\hline \hline Equipment & R Conf. & S Conf. & O Conf. \\
\hline EFIS-ND & 1.05318 & 1.06342 & 1.05440 \\
EFIS-PFD & 1.08161 & 1.08952 & 1.08302 \\
EFIS-ICP & 1.07183 & 1.08493 & 1.07405 \\
ADU & 1.21936 & 1.20554 & 1.22248 \\
IFC-IOP & 1.03095 & 1.04052 & 1.03157 \\
IFF & 1.20499 & 1.22881 & 1.20708 \\
MCDU & 1.05969 & 1.07249 & 1.06084 \\
IEDS-1A-1.2 & 1.13150 & 1.17981 & 1.13525 \\
IEDS-1A-3.4 & 1.05273 & 1.06378 & 1.05419 \\
IEDS-1B-1.2 & 1.06099 & 1.06232 & 1.06078 \\
IEDS-1B-3.4 & 1.03295 & 1.04377 & 1.03439 \\
CEU & 1.04570 & 1.04581 & 1.04500 \\
FECU & 1.04394 & 1.06713 & 1.04512 \\
\hline \hline
\end{tabular}

delay of the fully metal cockpit (R), and better than the delays found for the starting ESN (S).

The time-domain conclusions are corroborated by the $L$ values for the optimized configuration compared with those of the rest of the configurations analyzed (see Table VII). It can be
TABLE VII

L (IN H UNITS) FOR THE OPTIMIZED CONFIGURATION AND PERCENTAGES OF DifFERENCES With RESPECT TO PREVIOUS ONES

\begin{tabular}{lcccc}
\hline \hline Equipment & O Conf. & $\%(\mathrm{O}-\mathrm{R})$ & $\%(\mathrm{O}-\mathrm{S})$ & $\%(\mathrm{O}-\mathrm{E})$ \\
\hline EFIS-ND & $2.7687 \mathrm{E}-05$ & 5 & -18 & -4 \\
EFIS-PFD & $7.0352 \mathrm{E}-05$ & 4 & -36 & -2 \\
EFIS-ICP & $4.2211 \mathrm{E}-05$ & 7 & -26 & -7 \\
ADU & $1.6964 \mathrm{E}-04$ & 5 & -20 & -2 \\
IFC-IOP & $2.1548 \mathrm{E}-05$ & 1 & -16 & -2 \\
IFF & $2.1942 \mathrm{E}-04$ & 1 & -4 & -14 \\
MCDU & $3.1985 \mathrm{E}-05$ & 5 & -23 & -4 \\
IEDS-1A-1.2 & $2.1285 \mathrm{E}-04$ & 11 & -251 & -67 \\
IEDS-1A-3.4 & $2.6177 \mathrm{E}-05$ & 1 & -17 & -5 \\
IEDS-1B-1.2 & $6.7040 \mathrm{E}-05$ & 2 & -33 & -11 \\
IEDS-1B-3.4 & $2.1098 \mathrm{E}-05$ & 1 & -17 & -5 \\
CEU & $4.8688 \mathrm{E}-05$ & 0 & -8 & -7 \\
FECU & $4.1339 \mathrm{E}-05$ & 6 & -64 & -2 \\
\hline \hline
\end{tabular}

seen that the optimized configuration leads to smaller $L$ than the starting ESN for all the equipment. Its behavior is also better than the one provided by the more dense ESN analyzed, the so-called enhanced configuration, and similar to that supplied by the reference PEC case. These results demonstrate that an ESN with strips parallel to cable routes can be a good solution to the problem of current return on nonmetal structures.

\section{E. Combining Requirements: Final Solution $(F)$}

The configuration analyzed in the previous section yields results close to the ones provided by the PEC aerodynamic surfaces of the reference solution in most cases. However, a solution capable of being installed in the $\mathrm{A} / \mathrm{C}$ must meet all the requirements established, not only the initial ones, but also those found along the whole design phase by all the departments involved in it. In our case, additional final requirements affecting the ESN design included:

1) The forward cockpit skin needs to be metal to meet birdstrike requirements.

2) Some frames should also be metal to fulfill new structural requirements not taken into account at the beginning of this study.

3) Some lateral strips need to be removed in order to make easier the repair work needed to implement the ESN, which must be made, preferably, by reusing existing metal structure parts from the fuselage to minimize manufacturing efforts.

A final tradeoff configuration agreed upon by the different departments involved in the ESN design was proposed and analyzed in order to evaluate its efficiency. Fig. 9 shows the design while Table VIII lists the $L$ values calculated and compares them with the results from the rest of the configurations.

Table VIII shows a worsening with respect to the optimal solution. However, the deviations for this configuration, found as a compromise solution from the contributions of the different disciplines involved in the design process, have been considered acceptable, and it has been proposed as a proper candidate for the one that will be implemented in the carbon-fiber cockpit of a GRA. 


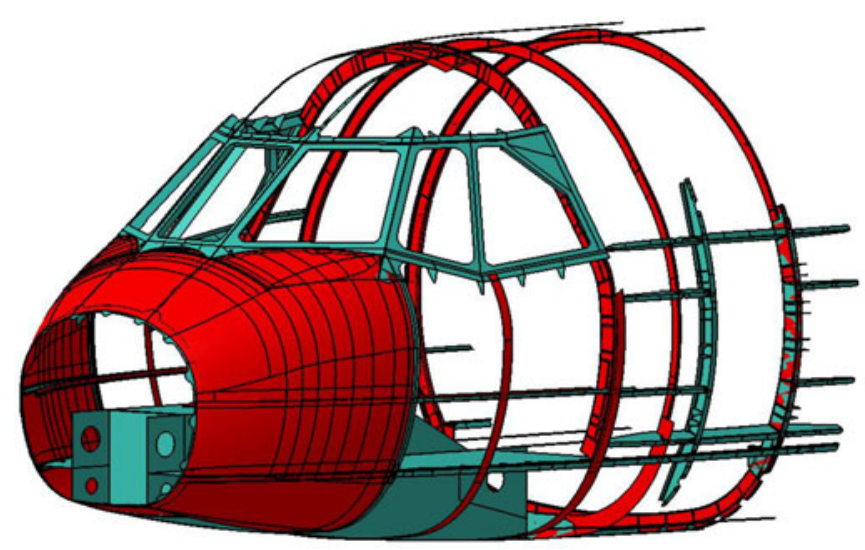

Fig. 9. Model with the final solution reached by different disciplines.

TABLE VIII

L (IN H UNITS) For THE FINAL SOLUTION AND PERCENTAGES OF DIFFERENCES With RESPECT TO PREVIOUS ONES

\begin{tabular}{lcccc}
\hline \hline Equipment & F Conf. & $\%(\mathrm{~F}-\mathrm{R})$ & $\%(\mathrm{~F}-\mathrm{S})$ & $\%(\mathrm{~F}-\mathrm{O})$ \\
\hline EFIS-ND & $2.8050 \mathrm{E}-05$ & 6 & -16 & 1 \\
EFIS-PFD & $6.5246 \mathrm{E}-05$ & -3 & -47 & -7 \\
EFIS-ICP & $4.2723 \mathrm{E}-05$ & 9 & -24 & 1 \\
ADU & $1.6847 \mathrm{E}-04$ & 4 & -21 & -1 \\
IFC-IOP & $2.1399 \mathrm{E}-05$ & 0 & -17 & -1 \\
IFF & $2.2628 \mathrm{E}-04$ & 4 & -1 & 3 \\
MCDU & $3.2445 \mathrm{E}-05$ & 6 & -21 & 1 \\
IEDS-1A-1.2 & $2.3770 \mathrm{E}-04$ & 24 & -214 & 12 \\
IEDS-1A-3.4 & $2.6657 \mathrm{E}-05$ & 3 & -15 & 2 \\
IEDS-1B-1.2 & $6.9854 \mathrm{E}-05$ & 7 & -28 & 4 \\
IEDS-1B-3.4 & $2.0604 \mathrm{E}-05$ & -2 & -20 & -2 \\
CEU & $5.3777 \mathrm{E}-05$ & 10 & -2 & 10 \\
FECU & $3.8179 \mathrm{E}-05$ & -2 & -77 & -8 \\
\hline \hline
\end{tabular}

\section{CONCLUSION}

In this paper, we describe the work done, inside the lowweight technology research within Clean Sky 1 project, to design an ESN with a distributed low-impedance metal network serving as current return path for power supplies, to prevent the impact on pieces of an electronic equipment of the different ground references, provided by the nonzero impedance of nonmetal structures.

We have shown that the graphical capabilities of simulation tools can help in the design of the ESN by identifying the current paths for the ideal PEC enclosure. From the surface current plots, we conclude that the addition of metal strips near to the cable routes, maintaining metal pieces in the structure closer to the cables, or even routing the cables along preexisting metal parts, lead to low inductances, thanks to the minimum loop area comprised by the ESN and the cables. This procedure has given results almost as good as those provided by the fully metal original $\mathrm{A} / \mathrm{C}$, in most cases.

We further shown that minor modifications of the optimized solution to taking into account the synergic task involving structural and systems designers have not significantly degraded the ESN behavior; thus, reaching a good solution in terms of both effectiveness of current returns and weight reduction.
Finally, though we have focused only on the equipment and power-line wiring, it should be noted that the ESN is also expected to contribute to the protection against lightning strikes, for being the primary voltage reference in an A/C carrying any functional and fault current as well as lightning currents, and thus also requiring electrical continuity between system components and the ESN [27]. Further work on this and other related topics are scheduled for the GRA within future research programs.

\section{REFERENCES}

[1] Clean Sky JTI project. (2008). [Online]. Available: http://www. cleansky.eu

[2] Military Handbook. Grounding, Bonding, and Shielding for Electronic Equipment and Facilities. Fort Belvoir, VA, USA: Defense Technical Information Center, Dec. 1987.

[3] Lightning and Surge Protection, Grounding, Bonding and Shielding Requirements for Facilities and Electronic Equipment, FAA Standard 019e, Dec. 2005.

[4] K. S. Yee, "Numerical solution of initial boundary value problems involving Maxwell's equations in isotropic media," IEEE Trans. Antennas Propag., vol. AP14, no. 3, pp. 302-307, May 1966.

[5] A. Taflove and S. C. Hagness, "Computational Electrodynamics: The Finite-Difference Time-Domain Method," 3rd ed. Boston, MA, USA: Artech House, 2005.

[6] "HIRF SE project. (2008). [Online]. Available: http://hirfse.axessim.eu/

[7] UGRFDTD EM solver. (2011). [Online]. Available: http://www. sembahome.org/

[8] R. Holland and L. Simpson, "Finite-difference analysis of EMP coupling to thin struts and wires," IEEE Trans. Electromagn. Compat., vol. EMC23, no. 2, pp. 88-97, May 1981.

[9] J. P. Bérenger, "A multiwire formalism for the FDTD method," IEEE Trans. Electromagn. Compat., vol. 42, no. 3, pp. 257-264, Aug. 2000.

[10] S. F. Romero, A. L. Morales, M. A. Cancela, and G. G. Gutierrez, "Validation procedure of low level coupling tests on real aircraft structure," in Proc. Int. Symp. Electromagn. Compat., Rome, Italy, 2012, pp. 1-6.

[11] G. G. Gutierrez, S. F. Romero, J. Alvarez, S. G. Garcia, and E. P. Gil, "On the use of FDTD for HIRF validation and certification," Progress Electromagn. Res. Lett., vol. 32, pp. 145-156, 2012.

[12] G. G. Gutierrez, J. Alvarez, E. Pascual-Gil, M. Bandinelli, R. Guidi, V. Martorelli, M. F. Pantoja, M. R. Cabello, and S. G. Garcia, "HIRF virtual testing on the C-295 aircraft: On the application of a pass/fail criterion and the FSV method," IEEE Trans. Electromagn. Compat., vol. 56, no. 4, pp. 854-863, Aug. 2014.

[13] ParaView. (2013). [Online]. Available: http://www.paraview.org/

[14] K. Johnson, P. Kogan, S. Feingold, N. Garrett, I. Guracar, W. Arendshorst, and P. Dayton, "Improving the quantitative ability of contrast enhanced ultrasound perfusion imaging: Effect of contrast administration rate and imaging plane orientation" in Proc. IEEE Int. Ultrasonics Symp., 2011, pp. 2436-2439. [Online]. Available: http://ieeexplore.ieee.org/stamp/ stamp.jsp?arnumber $=6293558$

[15] J. Alvarez, L. Angulo, M. Bandinelli, H. Bruns, M. Francavilla, S. Garcia, R. Guidi, G. Gutierrez, C. Jones, M. Kunze, J. Martinaud, I. Munteanu, M. Panitz, J. Parmantier, P. Pirinoli, Z. Reznicek, G. Salin, A. Schroder, P. Tobola, and F. Vipiana, "HIRF interaction with metal aircrafts. a comparison between TD and FD methods," in Proc. Int. Symp. Electromagn. Compat., 2012, pp. 1-6. [Online]. Available: http://ieeexplore.ieee.org/stamp/ stamp.jsp?arnumber $=6396703$

[16] J. Alvarez, L. Diaz Angulo, A. Rubio Bretones, M. Ruiz Cabello, and S. Garcia. (2013, Dec.). A leap-frog discontinuous galerkin time-domain method for HIRF assessment. IEEE Trans. Electromagn. Compat. [Online]. 55(6), pp. 1250-1259. Available: http://ieeexplore.ieee.org/stamp/ stamp.jsp?arnumber $=6530658$

[17] J. Alvarez, L. Angulo, A. Rubio-Bretones, and S. Gonzalez-Garcia, "Estimation of HIRF transfer functions by a leap-frog discontinuous Galerkin method" in Proc. Int. Symp. Electromagn. Compat., 2014, pp. 234-239. [Online]. Available: http://ieeexplore.ieee.org/stamp/ stamp.jsp?arnumber $=6930909$

[18] S. Dey and R. Mittra. (1997, Sep.). A locally conformal finite-difference time-domain (FDTD) algorithm for modeling three-dimensional perfectly conducting objects. IEEE Microw. Guided Wave Lett. [Online]. 
7(9). pp. 273-275. Available: http://ieeexplore.ieee.org/stamp/stamp.jsp? arnumber $=622536$

[19] S. G. Garcia, A. R. Bretones, B. G. Olmedo, and R. G. Martin, "Finite difference time domain methods," Time Domain Techniques in Computational Electromagnetics, D. Poljak Ed. Southampton, U.K.: WIT Press, 2003, pp. 91-132.

[20] M. Kuzuoglu and R. Mittra. (1996, Dec.). Frequency dependence of the constitutive parameters of causal perfectly matched anisotropic absorbers. IEEE Microw. Guided Wave Lett. [Online]. 6(12). pp. 447-449. Available: http://ieeexplore.ieee.org/stamp/stamp.jsp?arnumber $=544545$

[21] J. A. Roden and S. D. Gedney, "Convolution PML (CPML): An efficient FDTD implementation of the CFS-PML for arbitrary media," Microw. Opt. Technol. Lett., vol. 27, no. 5, pp. 334-339, Dec. 2000.

[22] Y. Wenhua, R. Mittra, T. Su, Y. Liu, and X. Yang, Parallel Finite-Difference Time-Domain Method (Artech House Electromagnetic Analysis Series). Boston, MA, USA: Artech House, 2006.

[23] G. G. Gutierrez, E. P. Gil, D. G. Gomez, and J. I. P. Gomez, "Finitedifference time-domain method applied to lightning simulation and aircraft certification process," in Proc. Int. Symp. Electromagn. Compat., York, U.K., 2011, pp. 750-755.

[24] Environmental Conditions and Test Procedures for Airborne Equipment, RTCA DO-160G, Washington, DC, USA, Dec. 2010.

[25] J. Grando, F. Issac, M. Lemistre, and J. Alliot, "Stability analysis including wires of arbitrary radius in FD-TD code," in Proc. Int Symp. Antennas and Propagation Soc., 1993, pp. 18-21. [Online]. Available: http://ieeexplore.ieee.org/stamp/stamp.jsp?arnumber $=385412$

[26] R. Serway and J. S. Faughn, College Physics, 5th ed. Philadelphia, PA, USA: Saunders, 1999

[27] F. A. Fisher, J. A. Plumer, and R. A. Perala, Aircraft Lightning Protection Handbook. Washington, DC, USA: Federal Aviation Administration, Sep. 1989

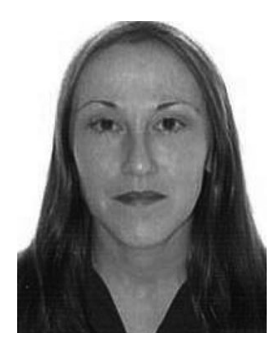

Guadalupe G. Gutierrez was born in Leon, Spain, in 1979. She received the M.Sc. degree in physics in 2002 from the University of Salamanca, Salamanca, Spain.

Since 2003, she has worked in different areas as EMC\&MW Engineer at Airbus Defence and Space. Currently, her main activities concern electromagnetic simulations on aircrafts.

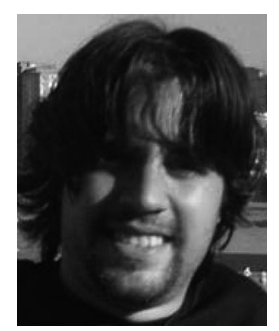

Daniel Mateos Romero was born in Huelva, Spain, in 1989. He received the B.Sc. and M.Sc. degrees in physics from the University of Granada, Granada, Spain, in 2012 and 2013, respectively, and since 2013, he has been working toward the Ph.D. degree at the Department of Electromagnetism and Matter Physics in physics working in time-domain solvers for electromagnetic compatibility applications.

$\mathrm{He}$ is part of the development team of the SEMBAUGRFDTD solver.

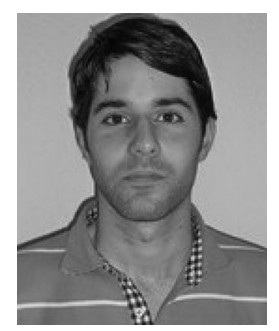

Miguel Ruiz Cabello was born in Granada, Spain $\mathrm{He}$ received the B.Sc. and M.Sc. degrees in physics from the University of Granada, Granada, Spain, in 2008 and 2010, respectively. Since 2010, he has been working toward the Ph.D. degree with the Department of Electromagnetism and Matter Physicsin physics working in HPC time-domain solvers for electromagnetic analysis and design.

He worked in the 7PM EU project HIRF-SE as a Developer of the UGRFDTD solver.

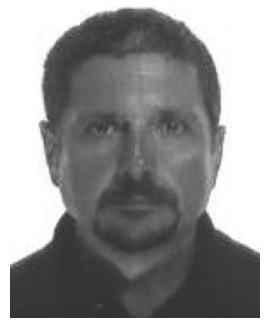

Enrique Pascual-Gil (M'12) was born in Madrid, Spain, in 1964. He received the M.Sc. degree in aeronautic and computer sciences from Universidad Politcnica de Madrid, Madrid, Spain, in 1985 and 1997, respectively.

He has been working from more than 25 years as an EMC\&MW Engineer in different areas. Since 2008, he has been the Head of computational electromagnetic at Airbus Military, Aragon, Spain, being responsible of the electromagnetic simulation team dedicated to numerical modeling of all electromagnetic aspects of an aircraft. Since 2011, he has been an Airbus EMC Expert. He has participated as a Technical Manager in various national and international technology projects. His current interests include the application of numerical methods to solve more complex cases in order to achieve a complete virtual testing for aircraft certification.

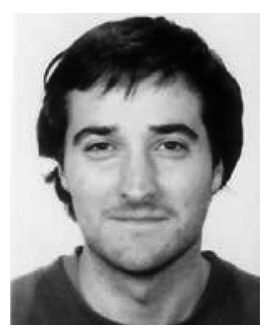

Luis Diaz Angulo was born in Basque Country, Spain, in 1985. He received the B.Sc. and M.Sc. degrees in physics from the University of Granada, Granada, Spain, in 2005 and 2007, respectively, and the Ph.D. degree from the same university in 2014, and the M.Sc. degree in electronics engineering, in 2015.

His research interests include time-domain numerical methods applied to electromagnetism, especially discontinuous Galerkin time-domain methods. Other interests include applications of numerical methods in terahertz technologies, GPR imaging, and bioelectromagnetics.

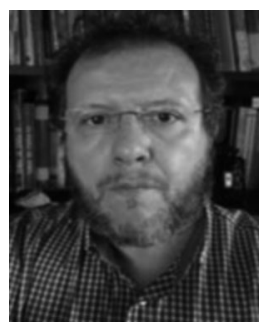

Salvador Gonzalez Garcia (SM'14) was born in 1966 in Baeza, Spain. He received the M.S. and Ph.D. degrees (with extraordinary award) in physics from the University of Granada, Granada, Spain, in 1989 and 1994, respectively.

In 1999, he joined the Department of Electromagnetism and Matter Physics, University of Granada as an Assistant Professor (qualified for a Full Professor since 2012). His current research interests include computational electromagnetics, electromagnetic compatibility, terahertz technologies, microwave imaging and sensing (GPR), bioelectromagnetics, and antenna design. He has published more than 70 refereed journal articles and book chapters, more than 80 conference papers and dozens of technical reports. He has participated and leaded several national and international projects with public and private funding.

Dr. Garcia received grants to stay as a Visiting Scholar at the University of Duisburg in 1997, the Institute of Mobile and Satellite Communication Techniques in 1998, the University of Wisconsin-Madison in 2001, and the University of Kentucky in 2005. 